\title{
Unsteady Free Convection in a Walter's-B Viscoelastic Flow past a Semi-Infinite Vertical Plate with Radiation and Chemical Reaction
}

\author{
J. Anand Rao ${ }^{1}$, P. Ramesh Babu ${ }^{2}$, and Sivaiah Sheri ${ }^{3}$ \\ ${ }^{1}$ Dept. of Mathematics, OsmaniaUniversity, Hyderabad, A.P., India. \\ ${ }^{2}$ Dept. of Mathematics, Sri Kottam Tulasi Reddy Memorial College of Engineering, Kondair(village), \\ Itikyala(Mandal), Mahabubnagar(Dist), A.P., India. \\ ${ }^{3}$ Dept. of Mathematics, Gitam University, Hyd, A.P., India.
}

\begin{abstract}
A numerical solution for the free convective, unsteady, laminar convective heat and mass transfer in a viscoelastic fluid along a semi-infinite vertical plate with radiation and chemical reaction is presented. The Walters-B liquid model is employed to simulate medical creams and other rheological liquids encountered in biotechnology and chemical engineering. This rheological model introduces supplementary terms into the momentum conservation equation. The dimensionless unsteady, coupled, and non-linear partial differential conservation equations for the boundary layer regime are solved by an efficient, accurate and unconditionally stable finite difference scheme of the Crank-Nicolson type. The velocity, temperature, and concentration fields have been studied for the effect of Prandtl number, viscoelasticity parameter, Schmidt number, radiation parameter, chemical reaction parameter and buoyancy parameters. The local skin-friction, Nusselt number and Sherwood number are also presented and analyzed graphically. It is observed that, when the viscoelasticity parameter increases, the velocity increases close to the plate surface. An increase in Schmidt number is observed to significantly decrease both velocity and concentration.
\end{abstract}

Key words: unsteady viscoelastic flow, semi-infinite vertical plate, Walters-B short-memory mode, finite difference method, radiation and chemical reaction.

\section{Introduction}

Heat and mass transfer in non-Newtonian fluids is of great interest in many operations in the chemical and process engineering industries including coaxial mixers [1], blood oxygenators [2], milk processing [3], steady-state tubular reactors, and capillary column inverse gas chromatography devices [4], mixing mechanisms [5], bubble-drop formation processes [6], dissolution processes [7], and cloud transport phenomena [8]. Many liquids possess complex shear-stress relationships which deviate significantly from the Newtonian (NavierStokes) model. External thermal convection flows in such fluids have been studied extensively using mathematical and numerical models and often employ boundary-layer theory. Many geometrical configurations have been addressed including flat plates, channels, cones, spheres, wedges, inclined planes, and wavy surfaces. Non-Newtonian heat transfer studies have included power-law fluid models [9-11] i. e. shear-thinning and shear thickening fluids, simple viscoelastic fluids [12, 13], Criminale-Ericksen-Fibley viscoelastic fluids [14], Johnson-Segalman rheological fluids [15], Bingham yield stress fluids [16], second grade (Reiner-Rivlin) viscoselastic fluids [17], third grade viscoelastic fluids [18], micropolar fluids [19], and bi-viscosity rheological fluids [20]. Viscoelastic properties can enhance or depress heat transfer rates, depending upon the kinematic characteristics of the flow field under consideration and the direction of heat transfer [21]. The Walters-B viscoelastic model [21] was developed to simulate viscous fluids possessing short memory elastic effects and can simulate accurately many complex polymeric, biotechnological, and tribological fluids. The Walters-B model has therefore been studied extensively in many flow problems. Soundalegkar and Puri [22] presented one of the first mathematical investigations for such a fluid considering the oscillatory two-dimensional viscoelastic flow along an infinite porous wall, showing that an increase in the Walters elasticity parameter and the frequency parameter reduces the phase of the skin-friction. Rath et al. [23] used a perturbation method to analyze the steady flow and heat transfer of Walters-B model viscoelastic liquid between two parallel uniformly porous disks rotating about a common axis, showing that drag is enhanced with suction but reduced with injection and that heat transfer rate is accentuated with a rise in wall suction or injection. Roy et al. [24] investigated heat transfer in Walters-B viscoelastic flow along a plane wall with periodic suction using a perturbation method including viscous dissipation effects. Raptis et al. [25] studied flat plate thermal convection boundary layer flow of a Walters-B fluid using numerical shooting quadrature. Chang et al. [26] analyzed the unsteady buoyancy-driven flow and species diffusion in a Walters-B viscoelastic flow along a vertical plate with transpiration effects. They showed that the flow is accelerated with a rise in viscoelasticity parameter with both 
time and distances close to the plate surface and that increasing Schmidt number suppresses both velocity and concentration in time whereas increasing species Grashof number (buoyancy parameter) accelerates flow through time. Chaudhary et al. [27] studied the Hall current and cross-flow effects on free and forced Walters-B viscoelastic convection flow with thermal radiative flux effects. Veena et al. [28] employed a perturbation and series expansion method and Kummer's functions to study two cases - prescribed power law surface temperature and power law surface heat flux. They showed that an increase in viscoelasticity increases surface shear stresses. Rajagopal et al. [29] obtained exact solutions for the combined non-similar hydromagnetic flow, heat, and mass transfer phenomena in a conducting viscoelastic Walters-B fluid percolating a porous regime adjacent to a stretching sheet with heat generation, viscous dissipation and wall mass flux effects, using confluent hypergeometric functions for different thermal boundary conditions at the wall.

Steady free convection heat and mass transfer flow of an incompressible viscous fluid past an infinite or semi-infinite vertical plate is studied since long because of its technological importance. Pohlhausen [30], Somers [31], and Mathers et al. [32] were the first to study it for a flow past a semi-infinite vertical plate by different methods. But the first systematic study of mass transfer effects on free convection flow past a semiinfinite vertical plate was presented by Gebhart et al. [33] who presented a similarity solution to this problem and introduced a parameter $N$ which is a measure of relative importance of chemical and thermal diffusion causing a density difference that drives the flow. Soundalgekar et al. [34] studied transient free convective flow past a semi-infinite vertical flat plate with mass transfer by using Crank-Nicolson finite difference method. In their analysis they observed that, an increase in $N$ leads to an increase in the velocity but a decrease in the temperature and concentration.

The study of heat and mass transfer with chemical reaction is of great practical importance to engineers and scientists because of its almost universal occurrence in many branches of science and engineering. Possible applications of this type of flow can be found in many industries like power industry and chemical process industries. Afify [35] studied the effects of radiation and chemical reaction on steady free convective flow and mass transfer of an optically dense viscous, incompressible and electrically conducting fluid past a vertical isothermal cone in the presence of a magnetic field. Recently, Rushi Kumar et.al. [36] have numerically analyzed the heat and mass transfer characteristics in the unsteady free convective flow of an incompressible viscoelastic fluid over a moving vertical cone and a flat plate in the presence of magnetic field and higher order chemical reaction.

Thermal radiation in fluid dynamics has become a significant branch of the engineering sciences and is an essential aspect of various scenarios in mechanical, aerospace, chemical, environmental, solar power, and hazards engineering. The viscous dissipation heat in the natural convective flow is important, when the flow field is of extreme size or at low temperature or in high gravitational field. Such effects are also important in geophysical flows and also in certain industrial operations and are usually characterized by the Eckert number. Whenever the temperature of surrounding fluid is high, the radiation effects play an important role and this situation does exist in space technology. In such cases one has to take into account the effects of radiation and free convection.

The interaction of radiation with laminar free convection heat transfer from a vertical plate was investigated by Cess [37] for an absorbing, emitting fluid in the optically thick region, using the singular perturbation technique. Arpaci [38] considered a similar problem in both the optically thin and optically thick regions and used approximate integral technique and first order profiles to solve the energy equation. Cheng et al [39] studied a related problem for an absorbing, emitting and isotropically scattering fluid, and treated the radiation part of the problem exactly with the normal mode expansion technique. Raptis [40] analyzed both the thermal radiation and free convection flow through a porous medium by using a perturbation technique. Hossain and Takhar [41] studied the radiation effects on mixed convection along a vertical plate with the uniform surface temperature using the Keller Box finite difference method. In all these papers, the flow taken steady, Mansour [42] studied the radiative and free convection effects on the oscillatory flow past a vertical plate. Raptis and Perdikis [43] considered the problem of thermal radiation and free convection flow past moving plate. Recently, Rajeswara Rao et. al. [44] studied the effect of radiation on unsteady free convection heat and mass transfer in a Walters-B viscoelastic flow past an impulsively started vertical plate.

\section{Constitutive equations for the Walters-B viscoelastic fluid}

Walters [21] has developed a physically accurate and mathematically amenable model for the rheological equation of state of a viscoelastic fluid of short memory. This model has been shown to capture the characteristics of actual viscoelastic polymer solutions, hydrocarbons, paints and other chemical engineering fluids. The Walters-B model generates highly non-linear flow equations which are an order higher than the classical Navier-Stokes (Newtonian) equations. It also incorporates elastic properties of the fluid which are important in extensional behavior of polymers. The constitute equations for a Walters-B liquid in tonsorial form may be presented as follows: 


$$
\begin{aligned}
& P_{i, k}=-P g_{i, j}+P^{*}{ }_{i, k} \\
& P_{i, j}^{*}=2 \int_{-\infty}^{t} \psi\left(t-t^{*}\right) e_{i, j}^{1}\left(t^{*}\right) d t^{*} \\
& \psi\left(t-t^{*}\right)=\int_{0}^{\infty} \frac{N(\tau)}{\tau} e^{-\left(t-t^{*}\right) / \tau} d \tau
\end{aligned}
$$

where $P_{i k}$ is the stress tensor, $p$ - the arbitrary isotropic pressure, $g_{t k}$ - the metric tensor of a fixed co-ordinate system $x_{i}, e_{i k}^{(1)}-$ the rate of strain tensor, and $N(t)$ - the distribution function of relaxation times, $\tau$. The following generalized form of eq. (1.2) has been shown by Walters [21] to be valid for all classes of motion and stress:

$$
P^{* i k}(x, t)=2 \int_{-\infty}^{t} \psi\left(t-t^{*}\right) \frac{\partial x^{i}}{\partial x^{* m}} \frac{\partial x^{k}}{\partial x^{* r}} e^{(1) m r}\left(x^{*}, t^{*}\right) d t^{*}
$$

in which $x_{i}^{*}=x_{i}^{*}\left(x, t, t^{*}\right)$ denotes the position at time $t^{*}$ of the element which is instantaneously at the position, $x_{i}$, at time, $t$. Liquids obeying the relations (1.1) and (1.4) are of the Walters-B' type. For such fluids with short memory i.e. low relaxation times, eq. (1.4) may be simplified to:

$$
P^{* i k}(x, t)=2 \eta_{0} e^{(1) i k}-2 k_{0} \frac{\partial e^{(1) i k}}{\partial t}
$$

in which $\eta_{o}=\int_{0}^{\infty} N(\tau) d \tau$ defines the limiting Walters-B' viscosity at low shear rates, $k_{0}=\int_{0}^{\infty} \tau N(\tau) d \tau$ is the Walters-B' viscoelasticity parameter and $\frac{\partial}{\partial t}$ is the convected time derivative. This rheological model is very versatile and robust and provides a relatively simple mathematical formulation which is easily incorporated into boundary layer theory for engineering applications [24, 25].

The aim of the present paper is to analyze the radiation effects on an unsteady two-dimensional laminar simultaneous free convection heat and mass transfer in a Walters -B viscoelastic flow past an impulsively started vertical plate in the presence of chemical reaction. The equations of continuity, linear momentum, energy and diffusion, which govern the flow field, are solved by using an implicit finite difference method of The Crank-Nicolson type. The behavior of the velocity, temperature, concentration, skin-friction and Sherwood number has been discussed for variations in the governing parameters. Here the results analyzed graphically.

\section{Mathematical Analysis}

An unsteady 2-D laminar free convective flow of a viscoelastic fluid past a semi-infinite vertical plate is considered. The $\mathrm{x}$-axis is taken along the plate in the upward direction and the $\mathrm{y}$-axis is taken normal to it. Initially, it is assumed that the plate and the fluid are at the same temperature $T_{\infty}^{\prime}$ and concentration $C_{\infty}^{\prime}$ level everywhere in the fluid. At time $t \geq 0$, Also, the temperature of the plate and the concentration level near the plate are raised to $T_{w}^{\prime}$ and $C_{w}^{\prime}$, respectively, and are maintained constantly thereafter. It is assumed that the concentration $C^{\prime}$ of the diffusing species in the binary mixture is very less in comparison to the other chemical species, which are present, and hence the Soret and Dufour effects are negligible. It is also assumed that there is no chemical reaction between the diffusing species and the fluid. Then, under the above assumptions, the governing boundary layer equations with Boussinesq's approximation are:

Continuity equation

$$
\frac{\partial u}{\partial x}+\frac{\partial v}{\partial y}=0
$$

Momentum equation

$$
\frac{\partial u}{\partial t^{\prime}}+u \frac{\partial u}{\partial x}+v \frac{\partial u}{\partial y}=g \beta\left(T^{\prime}-T_{\infty}^{\prime}\right)+g \beta^{*}\left(C^{\prime}-C_{\infty}^{\prime}\right)+v \frac{\partial^{2} u}{\partial y^{2}}-k_{0} \frac{\partial^{3} u}{\partial y^{2} \partial t^{\prime}}
$$

Energy equation 


$$
\frac{\partial T^{\prime}}{\partial t^{\prime}}+u \frac{\partial T^{\prime}}{\partial x}+v \frac{\partial T^{\prime}}{\partial y}=\alpha \frac{\partial^{2} T^{\prime}}{\partial y^{2}}-\frac{1}{\rho c_{p}} \frac{\partial q_{r}}{\partial y}
$$

Species equation

$$
\frac{\partial C^{\prime}}{\partial t^{\prime}}+u \frac{\partial C^{\prime}}{\partial x}+v \frac{\partial C^{\prime}}{\partial y}=D \frac{\partial^{2} C^{\prime}}{\partial y^{2}}-k_{1}\left(C^{\prime}-C_{\infty}^{\prime}\right)
$$

The initial and boundary conditions are

$$
\begin{aligned}
& t^{\prime} \leq 0: u=0, \quad v=0, \quad T^{\prime}=T_{\infty}^{\prime}, \quad C^{\prime}=C_{\infty}^{\prime} \\
& t^{\prime}>0: u=0, \quad v=0, \quad T^{\prime}=T_{w}^{\prime}, \quad C^{\prime}=C_{w}^{\prime} \quad \text { at } \quad y=0 \\
& u=0, v=0, T^{\prime}=T_{\infty}^{\prime}, C^{\prime}=C_{\infty}^{\prime} \quad \text { at } x=0 \\
& u \rightarrow 0, T^{\prime} \rightarrow T_{\infty}^{\prime}, \quad C^{\prime} \rightarrow C_{\infty}^{\prime} \quad \text { as } y \rightarrow \infty
\end{aligned}
$$

where $u$ and $v$ are velocity components in $\mathrm{x}$ - and $\mathrm{y}$-directions, respectively, $t^{\prime}$ is the time, $\mathrm{g}$ - the acceleration due to gravity, $\beta$ - the volumetric coefficient of thermal expansion, $\beta^{*}$ - the volumetric coefficient of expansion with concentration, $T^{\prime}-$ the temperature of the fluid in the boundary layer, $C^{\prime}-$ the species concentration in the boundary layer, $T_{w}^{\prime}$ - the wall temperature, $T_{\infty}^{\prime}$ - the free stream temperature far away from the plate, $C_{w}^{\prime}$ - the concentration at the plate, $C_{\infty}^{\prime}-$ the free stream concentration in fluid far away from the plate, $v$ - the kinematic viscosity, $\alpha$ - the thermal diffusivity, $\rho$ - the density of the fluid, $k_{1}$ - chemical reaction rate constant, and $D-$ the species diffusion coefficient.

By using the Rosseland approximation the radiative heat flux $q_{r}$ is given by

$$
q_{r}=-\frac{4 \sigma_{s}}{3 k_{e}} \frac{\partial T^{\prime 4}}{\partial y}
$$

Where $\sigma_{s}$ is the Stefan -Boltzmann constant and $k_{e}$ the mean absorption coefficient? It should be noted that by using the Rosseland approximation, the present analysis is limited to optically thick fluids. If temperature differences within the flow are significantly small, then equation [2.6] can be linearised by expanding $T^{\prime 4}$ into the Taylor series about $T_{\infty}^{\prime}$, which after neglect higher order terms takes the form:

$$
T^{\prime 4} \cong 4 T_{\infty}^{\prime 3} T^{\prime}-3 T_{\infty}^{\prime 4}
$$

In view of equations [2.6] and [2.7], eqn. [2.3] reduces to

$$
\frac{\partial T^{\prime}}{\partial t^{\prime}}+u \frac{\partial T^{\prime}}{\partial x}+v \frac{\partial T^{\prime}}{\partial y}=\alpha \frac{\partial^{2} T^{\prime}}{\partial y^{2}}+\frac{16 \sigma_{s} T_{\infty}^{\prime 3}}{3 k_{e} \rho c_{p}} \frac{\partial^{2} T^{\prime}}{\partial y^{2}}
$$

In order to write the governing equations and the boundary conditions in dimensionless form, the following non-dimensional quantities are introduced.

$$
\begin{aligned}
& X=\frac{x}{L}, Y=\frac{y G r^{1 / 4}}{L}, t=\frac{t^{\prime} v}{L^{2}} G r^{1 / 2}, U=\frac{u L G r^{-1 / 2}}{v}, V=\frac{v L G r^{-1 / 4}}{v} \\
& G r=\frac{g \beta L^{3}\left(T_{w}^{\prime}-T_{\infty}^{\prime}\right)}{v^{2}}, T=\frac{T^{\prime}-T_{\infty}^{\prime}}{T_{w}^{\prime}-T_{\infty}^{\prime}}, C=\frac{C^{\prime}-C_{\infty}^{\prime}}{C_{w}^{\prime}-C_{\infty}^{\prime}}, N=\frac{\beta^{*}\left(C_{w}^{\prime}-C_{\infty}^{\prime}\right)}{\beta\left(T_{w}^{\prime}-T_{\infty}^{\prime}\right)} \\
& \Gamma=\frac{k_{0} \sqrt{G r}}{L^{2}}, R=\frac{k_{e} k}{4 \sigma_{s} T_{\infty}^{\prime 3}}, \operatorname{Pr}=\frac{v}{\alpha}, S c=\frac{v}{D}, K r=\frac{k_{1} L^{2}}{v \sqrt{G r}}
\end{aligned}
$$

where $L$ is the characteristic length of the plate and k-the thermal conductivity.

Equations (2.1), (2.2), (2.8) and (2.4) are reduced to the following non-dimensional form

$$
\frac{\partial U}{\partial X}+\frac{\partial V}{\partial Y}=0
$$




$$
\begin{aligned}
& \frac{\partial U}{\partial t}+U \frac{\partial U}{\partial X}+V \frac{\partial U}{\partial Y}=\frac{\partial^{2} U}{\partial Y^{2}}+T+N C-\Gamma \frac{\partial^{3} U}{\partial Y^{2} \partial t} \\
& \frac{\partial T}{\partial t}+U \frac{\partial T}{\partial X}+V \frac{\partial T}{\partial Y}=\frac{1}{\operatorname{Pr}}\left(1+\frac{4}{3 R}\right) \frac{\partial^{2} T}{\partial Y^{2}} \\
& \frac{\partial C}{\partial t}+U \frac{\partial C}{\partial X}+V \frac{\partial C}{\partial Y}=\frac{1}{S c} \frac{\partial^{2} C}{\partial Y^{2}}-K r C
\end{aligned}
$$

The corresponding boundary conditions are

$$
\begin{array}{cc}
t \leq 0: U=0, V=0, T=0, C=0 & \\
t>0: U=0, V=0, T=1, C=1 & \text { at } \quad Y=0 \\
U=0, T=0, C=0 & \text { at } \quad X=0 \\
U \rightarrow 0, T \rightarrow 0, C \rightarrow 0 & \text { as } \quad Y \rightarrow \infty
\end{array}
$$

where $G r$ is the thermal Grashof number, $P r$ - the fluid Prandtl number, $S c$ - the Schmidt number, $N$ - the buoyancy ratio parameter, $R$ - radiation parameter and $\mathrm{Kr}$ - chemical reaction parameter.

To obtain an estimate of flow dynamics at the barrier boundary, we also define several important rate functions at $Y=0$. These are the dimensionless wall shear stress function, i. e. local skin friction function, the local Nusselt number (dimensionless temperature gradient) and the local Sherwood number (dimensionless species, i. e. contaminant transfer gradient) are computed with the following mathematical expressions [45]

$$
\tau_{X}=G r^{\frac{3}{4}}\left(\frac{\partial U}{\partial Y}\right)_{Y=0}, N u_{X}=\frac{-X G r^{\frac{3}{4}}\left(\frac{\partial T}{\partial Y}\right)_{Y=0}}{T_{Y=0}} \text { and } S h_{X}=\frac{-X G r^{\frac{3}{4}}\left(\frac{\partial C}{\partial Y}\right)_{Y=0}}{C_{Y=0}}
$$

We note that the dimensionless model defined by eqs. (2.10) to (2.13) under conditions (2.14) reduces to Newtonian flow in the case of vanishing viscoelasticity i.e. when $\Gamma \rightarrow 0$.

\section{Method Of Solution}

In order to solve these unsteady, non-linear coupled equations (2.10) to (2.13) under the conditions (2.14), an implicit finite difference scheme of Crank-Nicolson type has been employed. This method was originally developed for heat conduction problems [46]. The region of integration is considered as a rectangle with $X_{\max }=1$ and $Y_{\max }=14$ where $Y_{\max }$. corresponds to $Y=\infty$ which lies well outside the momentum, thermal, and concentration boundary layers. After some preliminary numerical experiments the mesh sizes have been fixed as $\mathrm{D} X=0.05, \mathrm{D} Y=0.25$ with time step $\mathrm{D} t=0.01$. The computations are executed initially by reducing the spatial mesh sizes by $50 \%$ in one direction, and later in both directions by $50 \%$ and the results are compared. It is observed that, in all the cases, the results differ only in the fifth decimal place. Hence these mesh sizes are considered to be appropriate mesh sizes for present calculations. The local truncation error in the finite difference approximation is $O\left(\mathrm{D} t^{2}+\mathrm{D} X+\mathrm{D} Y^{2}\right)$ and it tends to zero as $\mathrm{D} t, \mathrm{D} X$, and $\mathrm{D} Y$ tend to zero. Hence the scheme is compatible.

The finite difference equations corresponding to equations (2.7) - (2.10) are as follows

$$
\begin{aligned}
& \frac{\left[U_{i, j}^{n+1}-U_{i-1, j}^{n+1}+U_{i, j}^{n}-U_{i-1, j}^{n}+U_{i, j-1}^{n+1}-U_{i-1, j-1}^{n+1}+U_{i, j-1}^{n}-U_{i-1, j-1}^{n}\right]}{4 \Delta X}+\frac{\left[V_{i, j}^{n+1}-V_{i, j-1}^{n+1}+V_{i, j}^{n}-V_{i, j-1}^{n}\right]}{2 \Delta Y}=0 \\
& \frac{\left[U_{i, j}^{n+1}-U_{i, j}^{n}\right]}{\Delta t}+U_{i, j}^{n} \frac{\left[U_{i, j}^{n+1}-U_{i-1, j}^{n+1}+U_{i, j}^{n}-U_{i-1, j}^{n}\right]}{2 \Delta X}+V_{i, j}^{n} \frac{\left[U_{i, j+1}^{n+1}-U_{i, j-1}^{n+1}+U_{i, j+1}^{n}-U_{i, j-1}^{n}\right]}{4 \Delta Y}= \\
& \frac{\left[T_{i, j}^{n+1}+T_{i, j}^{n}\right]}{2}+N \frac{\left[C_{i, j}^{n+1}+C_{i, j}^{n}\right]}{2}+\frac{\left[U_{i, j-1}^{n+1}-2 U_{i, j}^{n+1}+U_{i, j+1}^{n+1}+U_{i, j-1}^{n}-2 U_{i, j}^{n}+U_{i, j+1}^{n}\right]}{2(\Delta Y)^{2}} \\
& -\Gamma \frac{\left[U_{i, j-1}^{n+1}-2 U_{i, j}^{n+1}+U_{i, j+1}^{n+1}+U_{i, j-1}^{n}-2 U_{i, j}^{n}+U_{i, j+1}^{n}\right]}{2(\Delta Y)^{2} \Delta t}
\end{aligned}
$$




$$
\begin{aligned}
& \frac{\left[T_{i, j}^{n+1}-T_{i, j}^{n}\right]}{\Delta t}+U_{i, j}^{n} \frac{\left[T_{i, j}^{n+1}-T_{i-1, j}^{n+1}+T_{i, j}^{n}-T_{i-1, j}^{n}\right]}{2 \Delta X}+V_{i, j}^{n} \frac{\left[T_{i, j+1}^{n+1}-T_{i, j-1}^{n+1}+T_{i, j+1}^{n}-T_{i, j-1}^{n}\right]}{4 \Delta Y}= \\
& \frac{1}{\operatorname{Pr}}\left(1+\frac{4}{3 R}\right) \frac{\left[T_{i, j-1}^{n+1}-2 T_{i, j}^{n+1}+T_{i, j+1}^{n+1}+T_{i, j-1}^{n}-2 T_{i, j}^{n}+T_{i, j+1}^{n}\right]}{2(\Delta Y)^{2}} \\
& \frac{\left[C_{i, j}^{n+1}-C_{i, j}^{n}\right]}{\Delta t}+U_{i, j}^{n} \frac{\left[C_{i, j}^{n+1}-C_{i-1, j}^{n+1}+C_{i, j}^{n}-C_{i-1, j}^{n}\right]}{2 \Delta X}+V_{i, j}^{n} \frac{\left[C_{i, j+1}^{n+1}-C_{i, j-1}^{n+1}+C_{i, j+1}^{n}-C_{i, j-1}^{n}\right]}{4 \Delta Y} \\
& =\frac{1}{S c} \frac{\left[C_{i, j-1}^{n+1}-2 C_{i, j}^{n+1}+C_{i, j+1}^{n+1}+C_{i, j-1}^{n}-2 C_{i, j}^{n}+C_{i, j+1}^{n}\right]}{2(\Delta Y)^{2}}-K r\left[\frac{C_{i, j}^{n+1}+C_{i, j}^{n}}{2}\right]
\end{aligned}
$$

Here, the subscript $i$-designates the grid point along the X-direction, $j$-along the $\mathrm{Y}$-direction and the superscript $n$ along the $t$-direction. An appropriate mesh size considered for the calculation is $\Delta X=0.05, \Delta Y=$ 0.25 , and the time step $\Delta t=0.01$.During any one time step, the coefficients $U_{i . j}^{n}$ and $V_{i . j}^{n}$ appearing in the difference equations are treated as constants. The values of $C, T, U$ and $V$ at time level $(n+1)$ using the known values at previous time level $(n)$ are calculated as follows.

The finite difference Equation (3.4) at every internal nodal point on a particular $i$-level constitute a tridiagonal system of equations. Such a system of equations is solved by using Thomas algorithm as discussed in Carnahan et al. [47]. Thus, the values of $C$ are known at every internal nodal point on a particular $i$ at $(n+1)^{\text {th }}$ time level. Similarly, the values of $T$ are calculated from Equation (3.3). Using the values of $C$ and $T$ at $(n+1)^{\text {th }}$ time level in Equation (3.2), the values of $U$ at $(n+1)^{\text {th }}$ time level are found in similar manner. Thus the values of $\mathrm{C}, \mathrm{T}$ and $\mathrm{U}$ are known on a particular i-level. Then the values of $V$ are calculated explicitly using the Equation (3.1) at every nodal point at particular $i$-level at $(n+1)^{\text {th }}$ time level. This process is repeated for various $i$-levels. Thus the values of $C, T, U$ and $V$ are known, at all grid points in the rectangular region at $(n+1)^{\text {th }}$ time level.

Computations are carried out until the steady state is reached. The steady-state solution is assumed to have been reached, when the absolute difference between the values of $U$ as well as temperature $T$ and concentration $C$ at two consecutive time steps are less than $10^{-5}$ at all grid points.

The truncation error in the finite difference approximation is $O\left(\Delta t^{2}+\Delta Y^{2}+\Delta X\right)$ and it tends to zero as $\Delta t$, $\Delta Y$ and $\Delta X$ tend to zero. Hence the scheme is compatible. The stability and compatibility ensure convergence. The derivatives involved in equation (2.15) are evaluated using five-point approximation formula and then the integrals are evaluated using Newton-Cotes closed integration formula.

\section{Results And Discussion}

A representative set of numerical results is shown graphically in Figures, to illustrate the influence of physical parameters, viz., viscoelasticity parameter $(\Gamma)=0.005$, buoyancy ratio parameter $(\mathrm{N})=1.0$, Schmidt number $(\mathrm{Sc})=0.24$ (low weight diffusing gas species), radiation parameter $(\mathrm{R})=0.5$ and Prandtl number $(\mathrm{Pr})=$ 0.71 (water-based solvents). All graphs therefore correspond to these values unless specifically otherwise indicated.

We have presented the variation of velocity $(U)$, temperature function $(T)$ and concentration $(C)$ versus $(Y)$ with on viscoelasticity $(\Gamma)$ at $X=1.0$. An increase in from 0 to $0.003,0.005$ and the maximum value of 0.007 , as depicted in fig. 1(a), clearly enhances the velocity, $U$ which ascends sharply and peaks in close vicinity to the plate surface $(Y=0)$. With increasing distance from the plate wall however the velocity $U$ is adversely affected by increasing viscoelasticity i.e. the flow is decelerated. The switchover in behavior corresponds to approximately $Y=1$. With increasing $(\mathrm{Y})$ velocity profiles decay smoothly to zero in the free stream at the edge of the boundary layer. The opposite effect is caused by an increase in time. A rise in $t$ from 3.36 through 3.38 , 3.39 to 3.42 causes a decrease in flow velocity, $\mathrm{U}$ nearer the wall in this case the maximum velocity arises for the least time progressed. fig. 1(b) increasing viscoelasticity is seen to decrease temperature throughout the boundary layer. The graphs show therefore that increasing viscoelasticity cools the flow. With progression of time, however the temperature, $T$ is consistently enhanced i.e. the fluid is heated as time progresses. A similar response is observed for the concentration field, $C$, in fig. 1(c), Increasing viscoelasticity again reduces concentration, showing that species diffuses more effectively in Newtonian fluids $(\Gamma=0)$ than in strongly viscoelastic fluids. Once again with greater elapse in time the concentration values are reduced throughout the boundary layer regime $(0<Y<14)$.

Fig.2 presents the effects of buoyancy ratio parameter $N$ on velocity profiles. The maximum time elapse to the steady-state scenario accompanies the only negative value of $N$ i. e. $N=-1.0$. For $N=0$ and then increasingly positive values of $N$ up to 10.0 , the time taken, $t$, is steadily reduced. As such the presence of aiding 
buoyancy forces (both thermal and species buoyancy force acting in unison) serves to stabilize the transient flow regime. The parameter $N=\beta^{*}\left(C_{w}^{\prime}-C_{\infty}^{\prime}\right) / \beta\left(T_{w}^{\prime}-T_{\infty}^{\prime}\right)$ and expresses the ratio of the species (mass diffusion) buoyancy force to the thermal (heat diffusion) buoyancy force. When $N=0$ the species buoyancy term, $N$ vanishes and the momentum boundary layer eq. (13) is de-coupled from the species diffusion (concentration) boundary layer eq. (15). Thermal buoyancy does not vanish in the momentum eq. (13) since the term $T$ is not affected by the buoyancy ratio. When $N<0$ we have the case of opposing buoyancy. An increase in $N$ from -1.0 , through $0,1,5$ to 10 clearly accelerates the flow $i$. $e$. induces a strong escalation in stream wise velocity $U$, close to the wall; thereafter velocities decay to zero in the free stream. At some distance from the plate surface, approximately $Y=2.0$, there is a cross-over in profiles. Prior to this location the above trends are apparent. However after this point, increasingly positive $N$ values in fact decelerate the flow. Therefore further from the plate surface, negative $N i$. e. opposing buoyancy is beneficial to the flow regime whereas closer to the plate surface it has a retarding effect.

To illustrate the effect of Prandtl number, $\operatorname{Pr}$ and time, $\mathrm{t}$ on velocity $\mathrm{U}$, temperature $\mathrm{T}$, and concentration C. Increasing Pr clearly reduces strongly velocity, $U$ [figure 3(a)] both in the near-wall regime and the far-field regime of the boundary layer. Velocity is therefore maximized when $\operatorname{Pr}=0.71$ (minimum) and minimized for the largest value of $\operatorname{Pr}$ (7.1). $\operatorname{Pr}$ defines the ratio of momentum diffusivity $(v)$ to thermal diffusivity. $\operatorname{Pr}<1$ physically corresponds to cases where heat diffuses faster than momentum. $\operatorname{Pr}=0.71$ is representative of water-based solvents and $P r>>1$. An increase in time, $\mathrm{t}$, also serves to strongly retard the flow. With increasing $P r$ from 0.71 through 3.0, 5.0 to 7.1, temperature, $T$ as shown in fig. 3(b), is markedly reduced throughout the boundary layer. Our computations show that a rise in $\operatorname{Pr}$ depresses the temperature function. Conversely the concentration values [figure 3(c)] are slightly increased with a rise in $\operatorname{Pr}$ from 0.71 through intermediate values to 7.1. However with progression of time the concentration is found to be decreased in the boundary layer regime.

It is of great interest to show how the radiation-conduction interaction effects on velocity, temperature and concentration. It is observed that, initially for lower values of the radiation parameter $(\mathrm{R})$, the heat transfer is dominated by conduction, as the values of $\mathrm{R}$ increases the radiation absorption in boundary layer decreases. i.e., an increase in the radiation parameter from 0.1 through $0.5,1.0,3.0$ and 5.0 results in a increase in the temperature and decrease in concentration shown in figs. 4(b) and 4(c). Here the velocity profiles increases initially and then slowly decreases to zero due to viscoelasticity shown in fig. 4(a).

The distributions of concentration $(C)$ versus coordinate $(Y)$ for various Schmidt numbers $(S c)$ and time $(t)$, close to the leading edge at $X=1.0$, is shown in Fig.5. These figures again correspond to a viscoelasticity parameter $\Gamma=0.005$ i.e weak elasticity and strongly viscous effects. An increase in Sc from 0.24 (low weight diffusing gas species) through 0.62 (oxygen diffusing) to 0.72 (denser hydrocarbon derivatives as the diffusing species), and 2.62, clearly strongly decelerates the flow. Figure 5 shows that increase in Schmidt number effectively depresses concentration values in the boundary layer regime since higher $S c$ values will physically manifest in a decrease of molecular diffusivity $(D)$ of the viscoelastic fluid. The effect of chemical reaction parameter $(\mathrm{Kr})$ is shown in Fig.6, and is seen to decrease with a rise in chemical reaction parameter.

In figs. 7(a) to (c) the variation of dimensionless local skin friction (surface shear stress) $\tau_{x}$, Nusselt number (surface heat transfer gradient) $\mathrm{Nu}_{x}$, and the Sherwood number (surface concentration gradient) $\left(\mathrm{Sh}_{\mathrm{x}}\right)$, $v s$. axial co-ordinate $(X)$ for various viscoelasticity parameters $(\Gamma)$ and time $(t)$ are illustrated Shear stress is clearly enhanced with increasing radiation and viscoelasticity (i.e. stronger elastic effects) $i . e$. the flow is accelerated, a trend consistent with our earlier computations in fig. 1(a). The ascent in shear stress is very rapid from the leading edge $(X=0)$ but more gradual as we progress along the plate surface away from the plate. With an increase in time, $t$, shear stress $\tau_{\mathrm{x}}$ is however increased. Increasing viscoelasticity $(\Gamma)$ is observed in fig. $7(\mathrm{~b})$ to enhance local Nusselt number $\mathrm{Nu}_{\mathrm{x}}$, values whereas they are again increased with greater time. Similarly in fig. 7(c) the local Sherwood number $\mathrm{Sh}_{x}$ values are elevated with an increase in viscoelastic effects $i . e$. a rise in from $\Gamma \rightarrow 0$ (Newtonian flow) through $0.003,0.005$ to 0.007 but depressed slightly with time.

The influence of Radiation parameter (R) and time (t) on $\tau_{x}, N u_{x}$ and $S h_{x}$, versus axial coordinate $(X)$ are depicted in figs. 8 (a)-(c). An increase in R from 0.1 through $0.5,1,3$ to 5 , strongly decreases both $\tau_{x}$ and $S h_{x}$ along the entire plate surface i.e. for all X. However with an increase in time $(t)$ both shear stress and local Sherwood number are reduced (from 8 (a) \& 8(b)). With increasing $R$ values, local Sherwood number, $N u_{x}$, as shown in figure 8(c), is boosted considerably along the plate surface; gradients of the profiles are also found to diverge with increasing $X$ values. However an increase in time $t$, and serves to enhance local Nusselt number. 


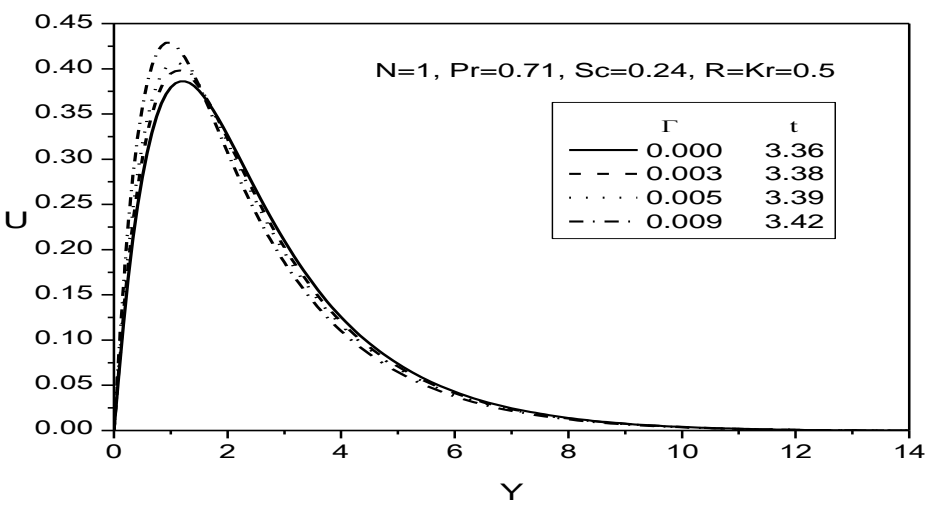

Fig.1(a). Steady state velocity profiles for different values of $\Gamma$

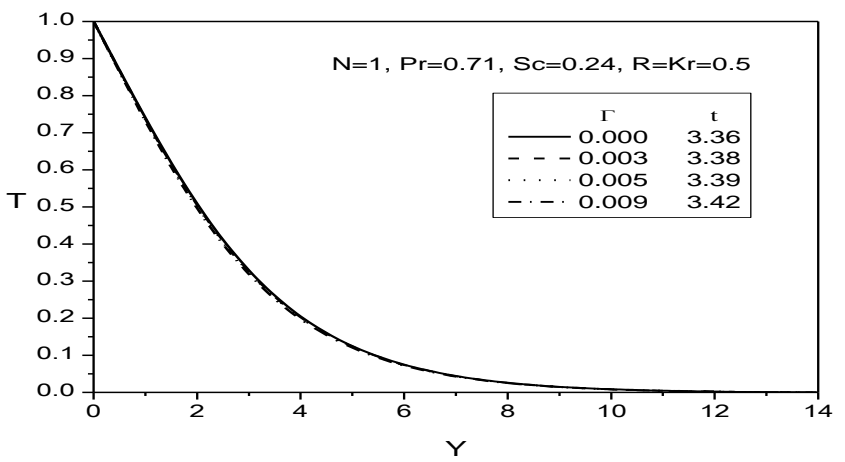

Fig.1(b). Steady state temperature profiles for different values of $\Gamma$

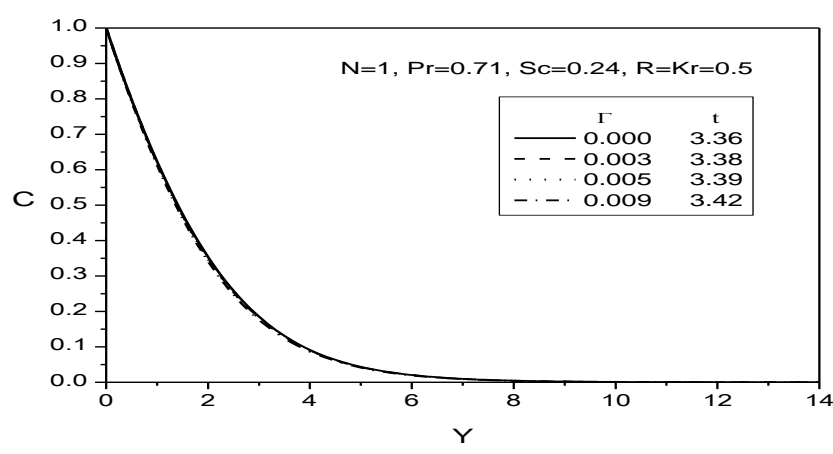

Fig.1(c). Steady state concentration profiles for different values of $\Gamma$

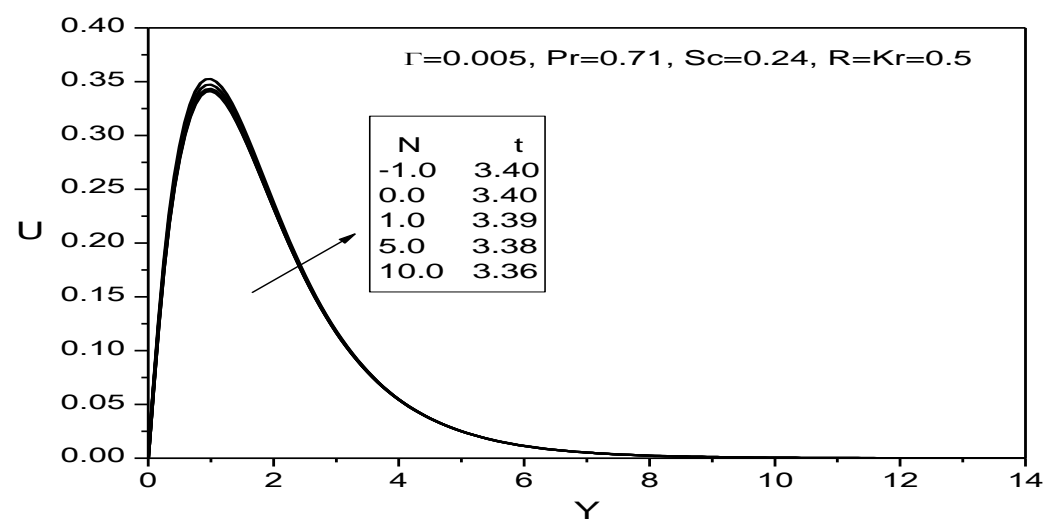

Fig.2. Steady state velocity profiles for different values of $\mathbf{N}$ 


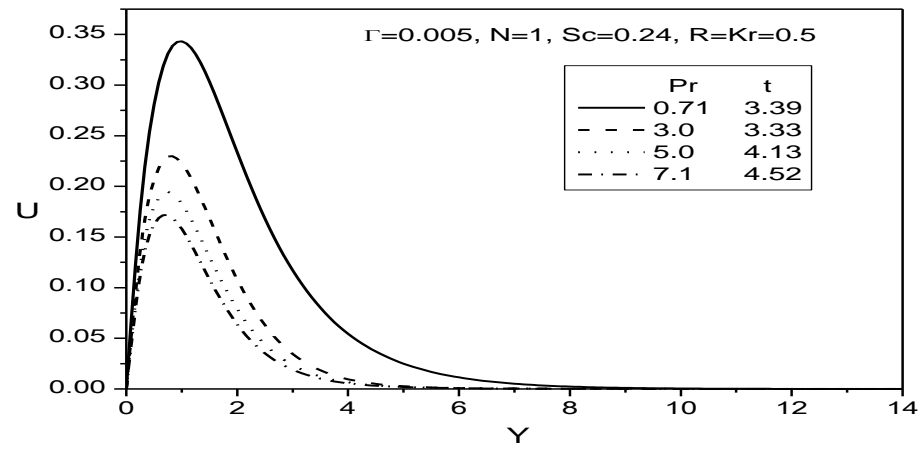

Fig.3(a). Steady state velocity profiles for different values of $\mathrm{Pr}$

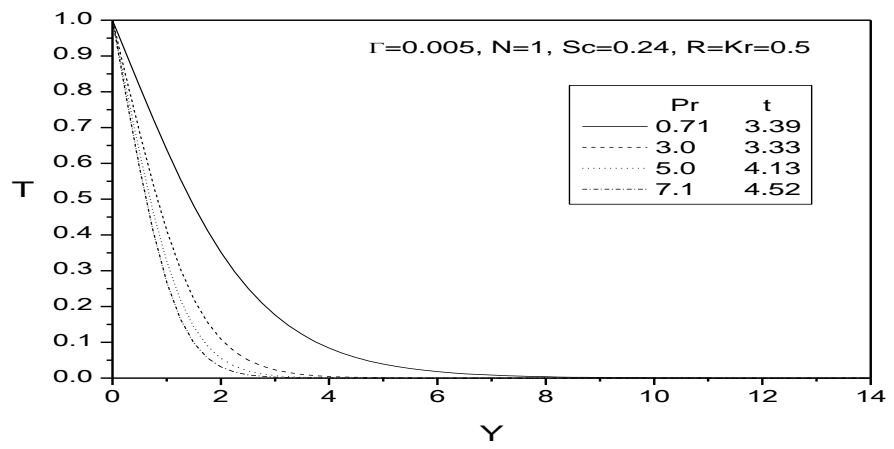

Fig.3(b). Steady state temperature profiles for different values of Pr

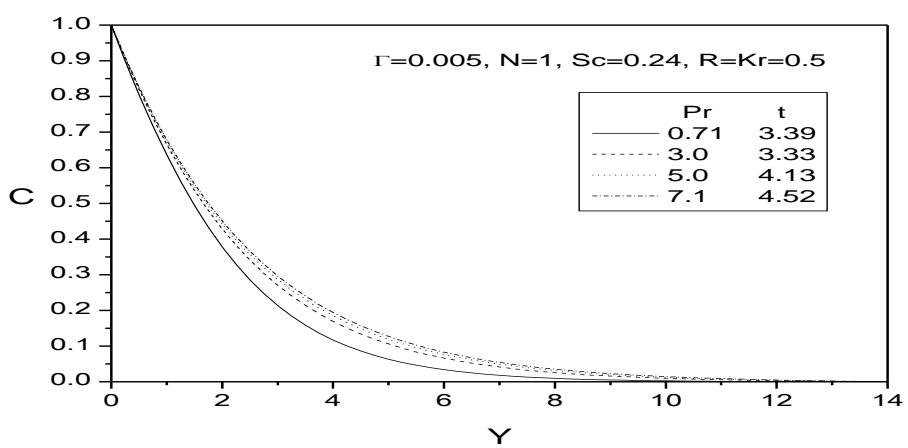

Fig.3(c). Steady state concentration profiles for different values of $\mathrm{Pr}$

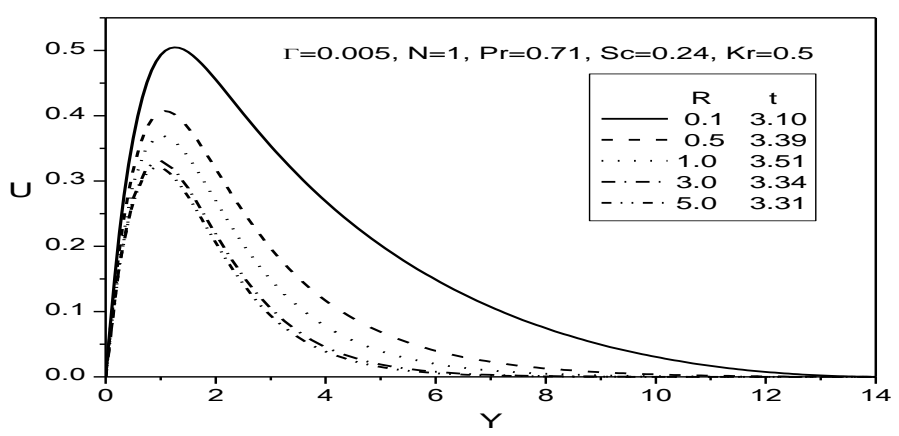

Fig.4(a). Steady state velocity profiles for different values of $R$ 


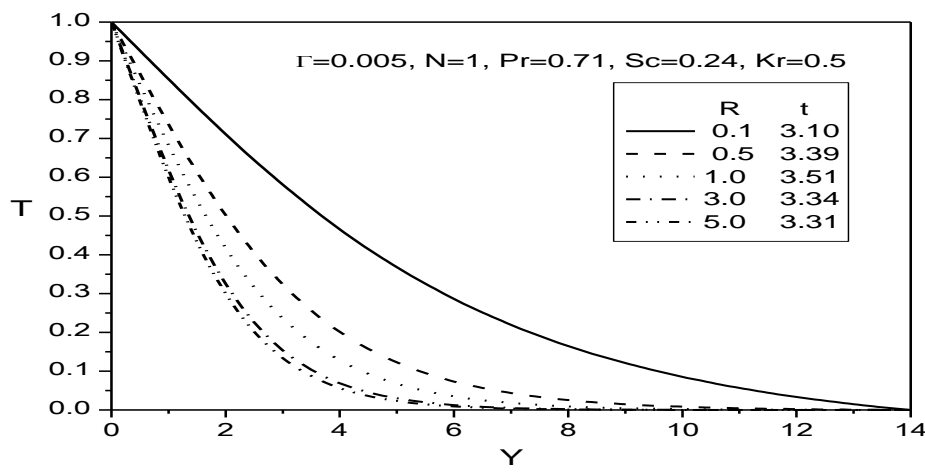

Fig.4(b). Steady state temperature profiles for different values of $R$

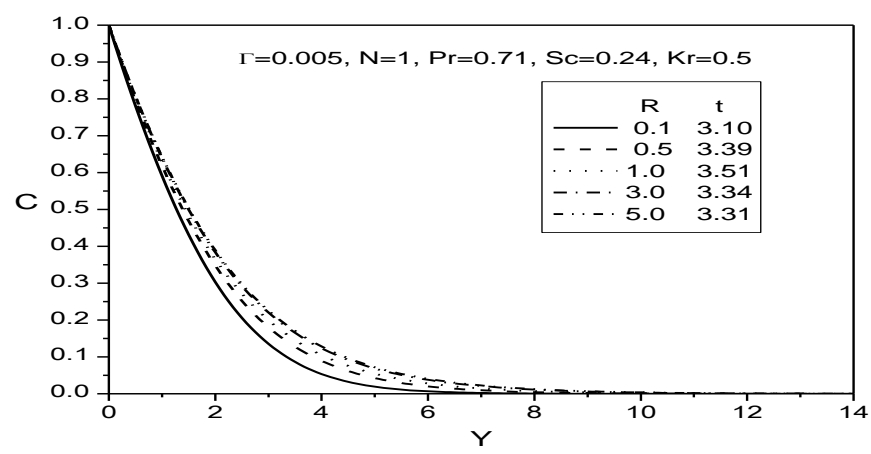

Fig.4(c). Steady state concentration profiles for different values of $R$

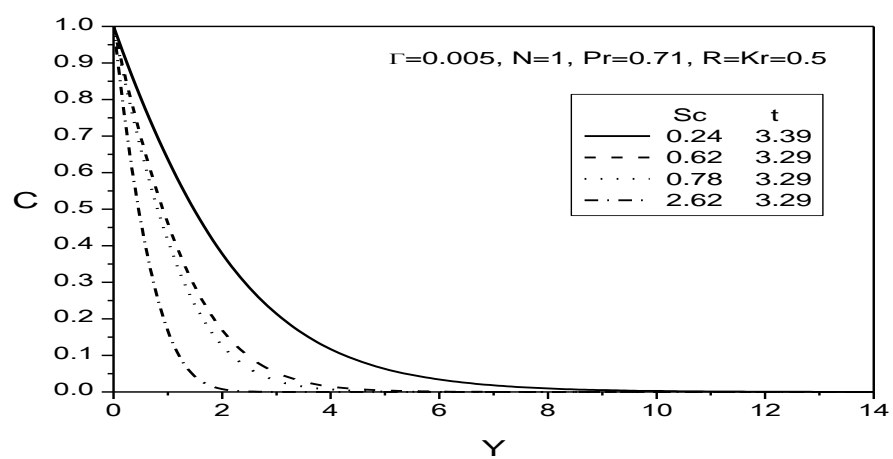

Fig.5. Steady state concentration profiles for different values of Sc

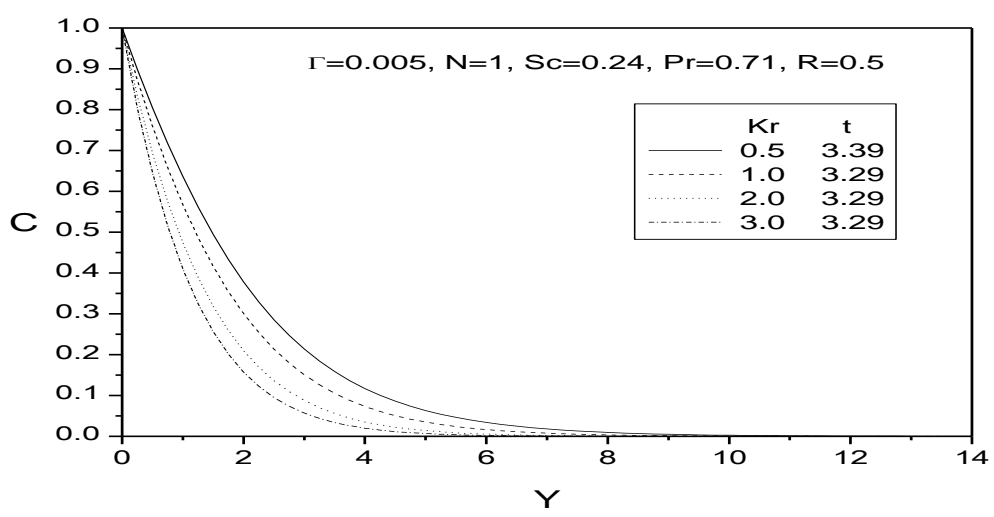

Fig.6. Steady state concentration profiles for different values of $\mathbf{K r}$ 


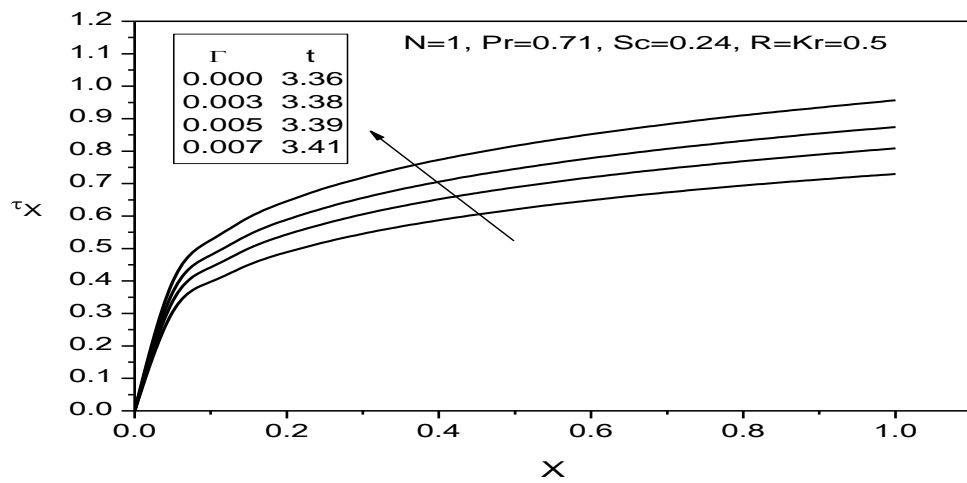

Fig.7(a). Local skin friction for different $\Gamma$

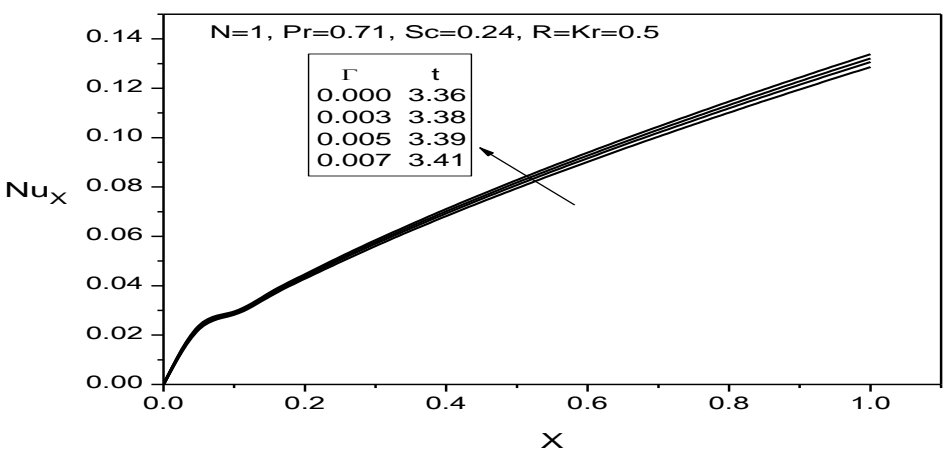

Fig.7(b). Local Nusselt number for different $\Gamma$

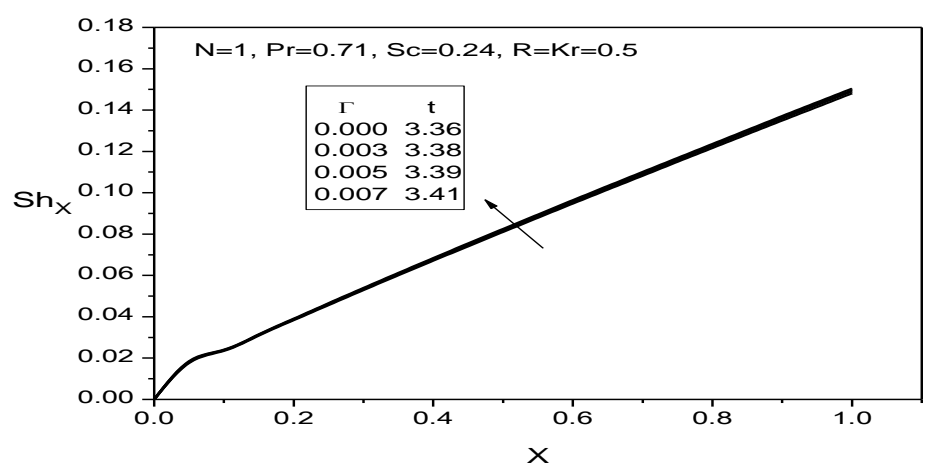

Fig.7(c). Local Sherwood number for different $\Gamma$

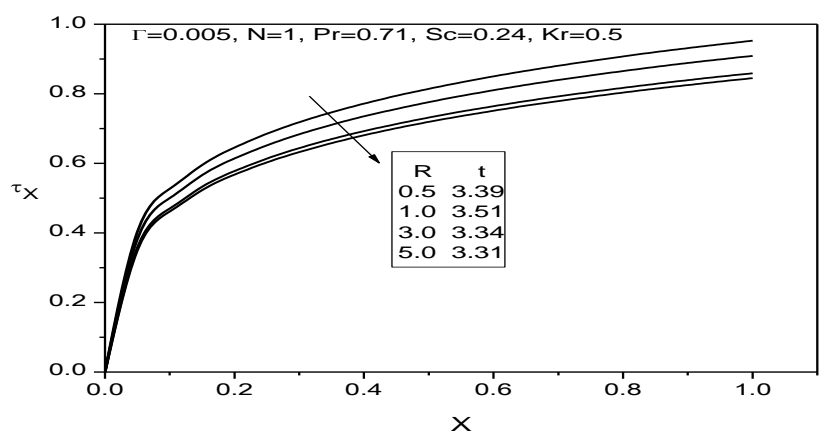

Fig.8(a). Local skin friction for different $R$ 


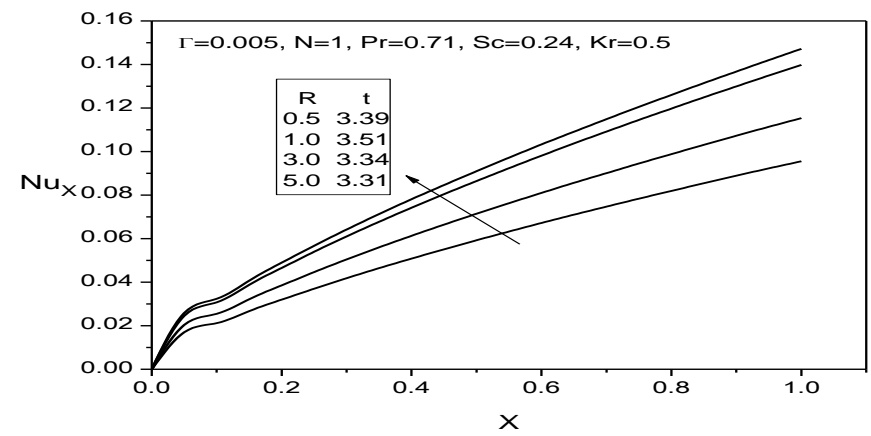

Fig.8(b). Local Nusselt number for different $R$

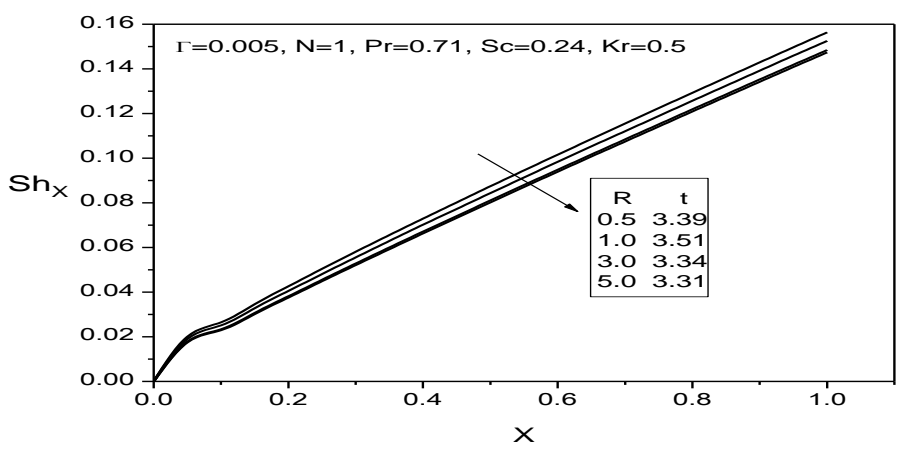

Fig.8(c). Local Sherwood number for different $R$

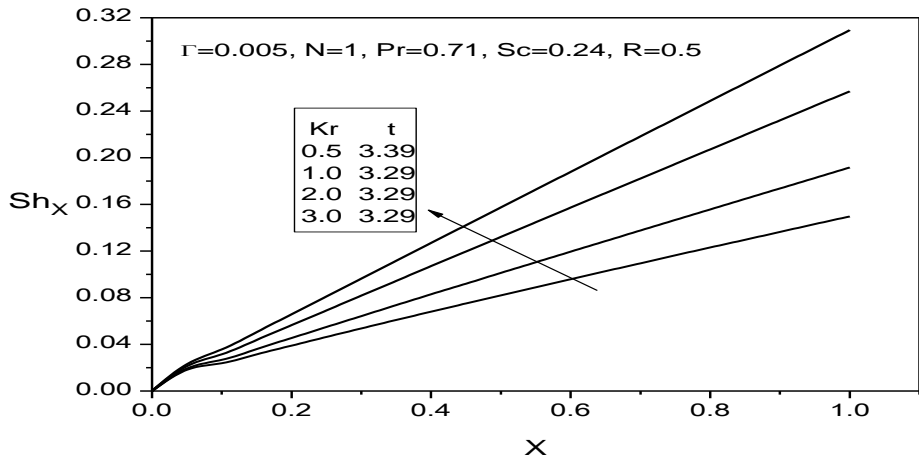

Fig.9. Local Sherwood number for different $K r$

\section{Conclusions}

A two-dimensional, unsteady laminar incompressible boundary layer model has been presented for the external flow, heat and mass transfer in a Walters-B viscoelastic buoyancy-driven radiative and chemically reactive flow from an impulsively started vertical plate. The Walters-B viscoelastic model has been employed which is valid for short memory polymeric fluids. The dimensionless conservation equations have been solved with the well-tested, robust, highly efficient, implicit Crank Nicolson finite difference numerical method. The present computations have shown that increasing viscoelasticity enhances shear stress (local skin friction), local Nusselt number and local Sherwood number, but reduces temperature and concentration in the boundary layer. Here the radiation effects on flow, initially for lower values of the radiation parameter, the heat transfer is dominated by conduction, as the values of $\mathrm{R}$ increases the radiation absorption in boundary layer increases. i.e., an increase in the radiation parameter results, decrease in the temperature and increase in concentration. This phenomenon is of interest in very high temperature (e.g. glass) flows in the mechanical and chemical process industries and is currently under investigation. Finally, the present computations have shown that increasing chemical reaction parameter enhances local Sherwood number, but reduces concentration in the boundary layer. 


\section{References}

[1]. Thibault, F., Tanguy, P. A., Power-Draw Analysis of a Coaxial Mixer with Newtonian and Non-Newtonian Fluids in the Laminar Regime, Chemical Engineering Science, 57 (2002), 18, pp. 3861-3872

[2]. Goerke, A. R., Leung, J., Wickramasinghe, S. R., Mass and Momentum Transfer in Blood Oxygenators, Chemical Engineering Science, 57 (2002), 11, pp. 2035-2046

[3]. Wang, W., Chen, G., Heat and Mass Transfer Model of Dielectric-Material-Assisted Microwave Freeze-Drying of Skim Milk with Hygroscopic Effect, Chemical Engineering Science, 60 (2005), 23, pp. 6542-6550

[4]. Vrentas, J. S., Vrentas, C. M., Axial Moment Analyses of Convective Heat and Mass Transfer Processes, Chemical Engineering Science, 51 (1996), 6, pp. 921-929

[5]. Ayazi Shamlou, P., Edwards, M. F., Heat Transfer to Viscous Newtonian and Non-Newtonian Fluids for Helical Ribbon Mixers, Chemical Engineering Science, 41 (1986), 8, pp. 1957-1967

[6]. Jarzebski, A. B., Malinowski, J. J., Transient Mass and Heat Transfer from Drops or Bubbles in Slow Non-Newtonian Flows, Chemical Engineering Science, 41 (1986), 10, pp. 2575-2578.

[7]. Elperin, T., Fominykh, A., Effect of Solute Concentration Level on the Rate of Coupled Mass and Heat Transfer During Solid Sphere Dissolution in a Uniform Fluid Flow, Chemical Engineering Science, 56 (2001), 10, pp. 3065-3074

[8]. Waslo, S., Gal-or, B., Boundary Layer Theory for Mass and Heat Transfer in Clouds of Moving Drops, Bubbles or Solid Particles, Chemical Engineering Science, 26 (1971), 6, pp. 829-838

[9]. Prakash Bharti, R., Chhabra, R. P., Eswaran, V., Effect of Blockage on Heat Transfer from a Cylinder to Power Law Liquids, Chemical Engineering Science, 62 (2007), 17, pp. 4729-4741.

[10]. Mahalingam, R., Chan, S. F., Coulson, J. M., Laminar Pseudoplastic Flow Heat Transfer with Prescribed Wall Heat Flux, Chemical Engineering J., 9 (1975), 2, pp. 161-166.

[11]. Kleinstreuer, C., Wang, T.-Y., Mixed Convection Heat and Surface Mass Transfer between Power-Law Fluids and Rotating Permeable Bodies, Chemical Engineering Science, 44 (1989), 12, pp. 2987-2994

[12]. White, J. L., Metzner, A. B., Thermodynamic and Heat Transport Considerations for Viscoelastic Fluids, Chemical Engineering Science, 20 (1965), 12, pp. 1055-1062

[13]. Shenoy, A. V., Mashelkar, R. A., Laminar Natural Convection Heat Transfer to a Viscoelastic Fluid, Chemical Engineering Science, 33 (1978), 6, pp. 769-776

[14]. Syrjala, S., Laminar Flow of Viscoelastic Fluids in Rectangular Ducts with Heat Transfer: A Finite Element Analysis, Int. Comm. Heat and Mass Transfer, 25 (1998), 2, pp. 191-204

[15]. Hayat, T., et al., Heat Transfer in Pipe Flow of a Johnson-Segalman Fluid, Int. Comm. Heat and Mass Transfer, 35 (2008), 10, pp. $1297-1301$

[16]. Hammad, K. J., Vradis, G. C., Viscous Dissipation and Heat Transfer in Pulsatile Flows of a Yield-Stress Fluid, Int. Comm. Heat and Mass Transfer, 23 (1996), 5, pp. 599-612

[17]. Beg, O. A., et al., Computational Fluid Dynamics Modelling of Buoyancy Induced Viscoelastic Flow in a Porous Medium with Magnetic Field, Int. J. Applied Mechanics Engineering, 6 (2001), 1, pp. 187-210

[18]. Bhargava, R., et al., Numerical Study of Heat Transfer of a Third Grade Viscoelastic Fluid in Non-Darcy Porous Media with Thermophysical Effects, Physica Scripta: Proc. Royal Swedish Academy of Sciences, 77 (2008), pp. 1-11.

[19]. Beg, O. A., et al., Computational Modeling of Biomagnetic Micropolar Blood Flow and Heat Transfer in a Two-Dimensional NonDarcian Porous Medium, Meccanica, 43 (2008), 4, pp. 391-410

[20]. Zueco, J., Beg, O. A., Network Numerical Simulation Applied to Pulsatile Non-Newtonian Flow through a Channel with Couple Stress and Wall Mass Flux Effects, Int. J. Applied Mathematics and Mechanics, 5 (2009), 2, pp. 1-16

[21]. Walters, K., Non-Newtonian Effects in Some Elastico-Viscous Liquids whose Behaviour at Small Rates of Shear is Characterized by a General Linear Equation of State, Quart. J. Mech. Applied. Math., 15 (1962), 1, pp. 63-76

[22]. Soundalgekar, V. M., Puri, P., On Fluctuating Flow of an Elastico-Viscous Fluid Past an Infinite Plate with Variable Suction, J. Fluid Mechanics, 35 (1969), 3, pp. 561-573

[23]. Rath, R. S., Bastia, S. N., Steady Flow and Heat Transfer in a Visco-Elastic Fluid between Two Coaxial Rotating Disks, Proc. Mathematical Sciences, 87 (1978), 8, pp. 227-236

[24]. Roy, J. S., Chaudhury, N. K., Heat Transfer by Laminar Flow of an Elastico-Viscous Liquid along a Plane Wall with Periodic Suction, Czech. J. Physics, 30 (1980), 11, pp. 1199-1209

[25]. Raptis, A. A., Takhar, H. S., Heat Transfer from Flow of an Elastico-Viscous Fluid, Int. Comm. Heat and Mass Transfer, 16 (1989), 2, pp. 193-197

[26]. Chang, T. B., et al., Numerical Study of Transient Free Convective Mass Transfer in a Walters-B Viscoelastic Flow with Wall Suction, J. Theoretical Applied Mechanics, under review (2009)

[27]. Chaudhary, R. C., Jain, P., Hall Effect on MHD Mixed Convection Flow of a Viscoelastic Fluid Past an Infinite Vertical Porous Plate with Mass Transfer and Radiation, Theoretical and Applied Mechanics, 33 (2006), 4, pp. 281-309.

[28]. Veena, P. H.,, Pravin, V. K., Padashetty, S. C., Non-Similar Solutions in Viscoelastic MHD Flow and Heat Transfer over a Porous Stretching Sheet with Internal Heat Generation and Stress Work, Int. J. Modern Mathematics, 2 (2007), 2, pp. 267-281

[29]. Rajagopal, K., Veena, P. H., Pravin, V. K., Nonsimilar Solutions for Heat and Mass Transfer Flow in an Electrically Conducting Viscoelastic Fluid over a Stretching Sheet Saturated in a Porous Medium with Suction/Blowing, J. Porous Media, 11 (2008), 2, pp. 219-230

[30]. Pohlhausen, E., Heat Transfer between Solid Body and Fluid Flow with Small Friction Forces and Small Heat Flux (in German), ZAMM, 1 (1921), pp. 115-121

[31]. Somers, E. V., Theoretical Considerations of Combined Thermal and Mass Transfer from Vertical Flat Plate, Journal of Appl. Mech., 23 (1956), 295-301

[32]. Mathers, W. G., Madden, A. J., Piret, E. L., Simultaneous Heat and Mass Transfer in Free Convection, Ind Engg. Chem., 49 (1956), 961-968

[33]. Gebhart, B., Pera, L., The Nature of Vertical Natural Convection Flows Resulting from the Combined Buoyancy Effects of Thermal and Mass Diffusion, International Journal of Heat and Mass Transfer, 14 (1971), 12, pp. 2025-2050

[34]. Soundalgekar, V. M., Ganesan, P., Finite Difference Analysis of Transient Free Convection with Mass Transfer on an Isothermal Vertical Flat Plate, International Journal of Engineering Science, 19 (1981), 6, pp. 757-770

[35]. Afify A.A., (2004), The effect of radiation on free convective flow and mass transfer past a vertical isothermal cone surface with chemical reaction in presence of transverse magnetic field, Canadian Journal of Physics, Vol.82, pp.447-458.

[36]. Rushi Kumar, B., and Sivaraj, R., (2013), Heat and mass transfer in MHD viscoelastic fluid flow over a vertical cone and flat plate with variable viscosity, International Journal of Heat and Mass Transfer, Vol.56, pp.370-379. 
[37]. Cess R .D., (1966), The interaction of thermal radiation with free convection heat and mass transfer. Int .J .of heat and mass transfer, Vol. 9, pp.1269- 1277.

[38]. Vedat S. Arpaci, (1968), effect if thermal radiation on the laminar free convection from a heatedvertical plate , Int. J. Heat and Mass Transfer, Vol. 11, pp.871-881.

[39]. Cheng E.H. and Ozisik M.N., (1972), Radiation with free convection in an absorbing, emitting and scattering medium. Int. J. Heat and Mass Transfer, Vol. 15, pp.1243-1252.

[40]. Raptis. A., (1998)., Radiation and free convection flow through a porous medium, Int.Commun. Heat and Mass transfer , Vol. 25, pp.289-295.

[41]. Hossain. M. A. And Takhar H.S., (1996), Radiation effect on mixed convection along a vertical plate with uniform surface temperature, Heat and mass transfer, Vol.31, pp. 243-248.

[42]. Mansour. M. H., (1990) Radiative and free convection effects on the oscillatory flow past a vertical plate. Astro physics and Space Science, Vol. 166, pp. 26-75.

[43]. Raptis .A. and Perdikis .C., (1999), Radiation and free convection flow past a moving plate. Appl. Mech.Eng., Vol. 4, pp. 817-821.

[44]. Rajeswara Rao, U., Ramachandra Prasad, V., Viswanath, G., Vasu, B., (2012), Radiation Effects on Unsteady Free Convection Heat and Mass Transfer in a Walters-B Viscoelastic Flow Past an Impulsively started Vertical Plate, International Journal of Scientific \& Engineering Research, Volume 3, Issue 8, pp.1-9.

[45]. Incropera, F. P., Dewitt, D. P., Fundamentals of Heat and Mass Transfer, 5th ed., John Wiley and Sons, New York, USA, 2007

[46]. Crank, J., Nicolson, P., A Practical Method for Numerical Evaluation of Solutions of Partial Differential Equations of the Heat Conduction Type, Proc. Camb. Phil. Society, 43 (1947), pp. 50-67

[47]. Carnahan B., Luther H.A. and Willkes J.O.(1969), Applied Numerical Methods, John Wiley \& Sons, New York. 